\title{
COMPARAÇÃO DO ESTADO MENTAL \\ DE IDOSOS COM DIFERENTES NIVEIS \\ EDUCACIONAIS INSERIDOS EM PRÁTICAS \\ DE ATIVIDADES FISICAS
}

\author{
Juliana Bayeux Dascal ${ }^{1}$ \\ Raquel de Melo Martins² \\ Inara Marques ${ }^{3}$ \\ Jean Curti ${ }^{4}$ \\ Denílson de Castro Teixeira ${ }^{5}$
}

resumo

O objetivo do estudo foi comparar a relação entre diferentes práticas de atividade física e o desempenho cognitivo de idosos. Participaram do mesmo 54 indivíduos idosos sadios, com 70,7 ( \pm 6,6) anos, subdivididos em 3 grupos: 18 idosos praticantes de caratê (GCT), 18 idosos praticantes de caminhadas sem supervisão de profissional (GCA) e 18 não praticantes de atividades físicas regulares (GNP). Foi aplicado o questionário Mini-Exame do Estado Mental (MEEM), associando-o com o período de tempo educacional e o envolvimento na prática de exercícios físicos. Os resultados mostraram que nas variáveis do MEEM, os idosos caratecas apresentaram desempenho significativamente melhor do que os idosos que praticam caminhadas e do que os idosos não treinados. Já no escore total do MEEM, foram encontradas correlações significativas nos grupos GCT e GCA. Dessa forma, conclui-se que idosos tendem a ter melhor estado mental, tanto pela influência dos anos de estudo, como pela prática de exercícios físicos.

palavras - chave

Cognição. Exercício. Envelhecimento.

1 Professora Doutora do Departamento de Educação Física da Universidade Estadual de Londrina. CEF/DEF/UEL. E-mail: jbdascal@yahoo.com.br

2 Graduada em bacharelado em Educação Física na Universidade Estadual de Londrina. Mestre em Desempenho motor e atividade física. CEF/DEF/UEL E-mail: quel_martins@hotmail.com

3 Professora Doutora do Departamento de Educação Física da Universidade Estadual de Londrina. E-mail: inaramarques@hotmail.com

4 Graduando do curso de bacharelado em Educação Física da Universidade Estadual de Londrina. E-mail: jean.curti@gmail.com

5 Professor Doutor do Departamento de Educação Física da Universidade Estadual de Londrina e Professor Doutor do Centro de Pesquisa em Ciências da Saúde da Universidade Norte do Paraná. E-mail: denict.9@gmail.com 
O processo de envelhecimento envolve diversas alterações tanto no desempenho motor quanto no cognitivo. Nesse sentido, o entendimento desses processos se faz necessário e imprescindível, frente ao aumento da expectativa de vida (PEREIRA et al., 2002) e à consequente manutenção da qualidade de vida de idosos.

Atualmente, um dos aspectos considerados fundamentais no processo de minimizar declínios relacionados ao envelhecimento é a prática regular de atividades físicas. Por meio desta, idosos podem atenuar degenerações motoras típicas dessa idade e até mesmo aproximar seus desempenhos dos de adultos jovens (SPIRDUSO, 1975; RIKLI; EDWARDS, 1991; STARKES et al., 1999; ZISI et al., 2001).

Além disso, estudos relacionando a prática de atividades físicas com o envelhecimento mostram que essas atividades também atenuam declínios relacionados à cognição. De acordo com Colcombe et al. (2003), as regiões cerebrais mais gravemente afetadas pelo envelhecimento são as que mais se beneficiam da prática regular de atividades físicas. Portanto, os seus efeitos relacionam-se não somente a variáveis comportamentais, mas também às estruturas funcionais, tais como velocidade de transmissão de estímulos ao SNC, aumentando a perfusão sanguínea cerebral e a oxigenação dos tecidos (ERICKSON et al., 2011; COLCOMBE et al. 2003).

Recentemente, estudos compararam os efeitos que a prática de atividades físicas pode exercer no desempenho cognitivo (KARIMOOY et al., 2012; PSALTOPOULOU et al., 2008). Para avaliar o desempenho cognitivo, os pesquisadores têm utilizado o Mini-Exame do Estado Mental (MEEM), que se caracteriza por uma escala de avaliação cognitiva cujo objetivo é auxiliar na investigação de possíveis déficits cognitivos em indivíduos de risco, como é o caso dos idosos.

Em um estudo transversal, no qual foi identificado que a prática de atividades físicas ao longo da vida influenciou positivamente o estado cognitivo de indivíduos adultos e idosos, Karimooy et al. (2012) compararam o resultado obtido por meio da aplicação do MEEM em indivíduos com mais de 55 anos, de ambos os sexos, com e sem histórico de práticas de atividades físicas ao longo da vida. Os resultados mostraram que os indivíduos de ambos os sexos, com passado esportivo ou de prática regular de atividades físicas, apresentaram melhor desempenho cognitivo do que os que não tiveram esse histórico de estilo de vida. Também foram identificados efeitos positivos da prática regular de atividades físicas sobre o desempenho cognitivo no estudo 
de Psaltopoulou et al. (2008), no qual os autores relacionaram o MEEM com diferentes variáveis, dentre elas dados antropométricos, sociodemográficos, nutricionais e de estilos de vida. Como resultado dessa investigação, observou-se, de maneira geral, que a boa condição nutricional, adquirida em fases iniciais da vida, e a prática regular de atividades físicas provocam um efeito de proteção à cognição.

Portanto, observa-se que a literatura tem ressaltado a existência de uma relação positiva entre a prática regular de atividades físicas não somente para aspectos motores, como também para o domínio cognitivo. Entretanto, ainda não foram totalmente investigados e esclarecidos aspectos relacionados ao tipo de atividade física e a sua intensidade e a relação desses tipos de prática com idosos brasileiros, assim como de outros aspectos que permeiam a prática de atividades físicas e que estejam relacionados à melhoria do desempenho cognitivo.

Um dos aspectos que queremos investigar em nosso estudo é se tipos diferenciados de práticas motoras podem interferir também de maneira diferenciada sobre o estado cognitivo de idosos. Mais especificamente, será que para atividades que envolvem estratégias de ataque e defesa, como o caratê, além de colocar o praticante sempre em condições de tomadas de decisões rápidas, a demanda cognitiva é maior e com isso poderá aprimorar o estado cognitivo de seus praticantes? Por outro lado, será que a prática da caminhada, a qual não demanda estratégias cognitivas e tomadas de decisões como no caratê, por preservar aspectos importantes para a cognição (como maior oxigenação cerebral, por exemplo) já é suficiente para a preservação do estado cognitivo de idosos?

Neste sentido, se a prática de atividades físicas for realmente um fator que pode influenciar sobre o estado cognitivo de idosos, nossos resultados mostrarão melhores desempenhos no MEEM para o grupo de idosos ativos. Entretanto, se os benefícios propiciados pela prática de atividades físicas influenciarem somente aspectos físicos e motores de idosos, não serão observadas diferenças entre os resultados do MEEM entre idosos ativos e sedentários. Ainda, se o tipo de prática investigado - caratê e caminhada - apresentarem efeitos diferenciados para o MEEM, ou seja, se os idosos caratecas apresentarem desempenhos superiores aos dos idosos que praticam caminhada, provavelmente o tipo de envolvimento cognitivo demandado pela prática do caratê (como por exemplo, estratégias de ataque e defesa) possa ser um fator que estimule a preservação cognitiva de idosos de maneira mais pronunciada do que somente a prática da caminhada. Por outro lado, se não verificarmos efeitos diferenciados para os dois tipos de prática investigados, 
poderemos inferir que somente a prática de atividades físicas por si só já é suficiente para a preservação cognitiva, independente de quais funções sejam estimuladas durante a prática.

Dessa maneira, nosso estudo teve como objetivo comparar e correlacionar o estado mental de idosos com diferentes níveis educacionais e praticantes de diferentes tipos de atividades físicas.

\subsection{Participantes}

Participaram deste estudo 54 indivíduos idosos sadios, com $70,7( \pm 6,6)$ anos, subdivididos em 3 grupos: 18 idosos (7 homens e 11 mulheres) praticantes de caratê (GCT), 18 idosos (5 homens e 13 mulheres) praticantes de caminhadas sem supervisão de profissional (GCA) e 18 idosos (9 homens e 9 mulheres) não praticantes de atividades físicas regulares (GNP).

Os idosos caratecas foram recrutados de uma academia de artes marciais do município de Londrina-PR que desenvolve aulas de caratê, sendo, os mesmos, alunos de uma turma composta, especificamente, por idosos. As aulas são realizadas regularmente três vezes por semana e são compostas de atividades envolvendo aquecimento articular e alongamentos, como parte inicial das aulas, e de movimentos particulares ao caratê, simulando golpes e movimentos dinâmicos, tanto com membros inferiores quanto superiores, como parte principal da aula. Já os idosos que participavam de caminhadas regulares realizam essas atividades sem acompanhamento de profissional de Educação Física, de três a cinco vezes por semana. Os idosos não praticantes de atividade física e os inseridos em atividade de caminhada foram recrutados nas Unidades Básicas de Saúde do Município de Londrina-PR.

Os critérios de seleção da amostra foram por conveniência, na medida em que os idosos caratecas foram inicialmente contatados e selecionados da academia de caratê e, a partir das características amostrais deste grupo, foram selecionados os outros grupos experimentais aqui investigados. Como critérios de inclusão no estudo, todos os idosos deveriam ser sadios, alfabetizados e sem doenças e/ou comprometimentos sensoriais e/ou motores que impedissem a realização do teste proposto. 
Todos os participantes, após terem sido informados os objetivos e procedimentos do estudo, foram convidados a assinar o Termo de Consentimento Livre e Esclarecido e a responder aos questionários utilizados neste estudo. Os procedimentos adotados na pesquisa obedeceram aos Critérios de Ética em Pesquisa com Seres Humanos, conforme resolução no 196/96 do Conselho Nacional de Saúde, tendo sido aprovado pelo Comitê de Ética da Instituição local sobre o parecer $n^{0}$ 287/2011.

\subsection{Instrumentos}

Para a realização deste estudo foi utilizado o MEEM (Folstein et al., 1975), adaptado para a população brasileira por Bertolucci et al. (1994). Como já esclarecido acima, o MEEM é um importante instrumento no rastreio de comprometimento cognitivo. Este questionário é composto por questões relacionadas à orientação temporal e espacial, memória imediata, cálculo, evocação, nomeação e repetição de palavras, comando de uma ação, leitura, elaboração de uma frase e cópia de um desenho.

O teste não é controlado pelo tempo de duração, mas pelo desempenho, cujo escore máximo é de 30 pontos, sendo pontuadas as respostas corretas. Nesse caso, a incapacidade de responder a um item é considerada como erro e, portanto, não pontuada. As notas de corte dependem do grau de escolaridade e, nesse estudo, seguiu-se a classificação proposta por Lourenço e Veras (2006), que apresenta pontos de corte para idosos analfabetos de 18/19 pontos (ausência/presença de comprometimento cognitivo) e para indivíduos escolarizados de 24/25 pontos (ausência/presença de comprometimento cognitivo). A aplicação do teste MEEM foi feita individualmente por pesquisadores previamente treinados e seus resultados foram analisados e comparados com valores referencias para o grupo etário aqui investigado.

Além da aplicação do MEEM, também foi aplicado um questionário sobre o período de tempo educacional (para todos os grupos), tendo em vista que a pontuação do MEEM está associada ao nível educacional do sujeito e de envolvimento na atividade física (somente para os grupos de caratecas e praticantes de caminhada). 
A aplicação dos questionários foi realizada individualmente por pesquisadores previamente treinados, a fim de garantir maior fidedignidade nas respostas e resultados. Para os grupos de idosos ativos, as entrevistas foram realizadas antes ou após a prática da atividade física dos mesmos, no próprio local de prática. Vale ressaltar que a maior dificuldade encontrada na aplicação dos questionários ocorreu para o grupo de caratecas, já que alguns deles foram alfabetizados no Japão e lá permaneceram durante algum tempo. Tal fato dificultou o tempo de compreensão dos comandos do avaliador na aplicação do MEEM, principalmente na repetição das palavras; entretanto, apesar do maior tempo de aplicação dos testes, a aplicação dos mesmos ocorreu de maneira similar aos outros grupos avaliados.

\subsection{Análise dos dados}

Os resultados descritivos foram apresentados pela mediana e intervalos interquartílicos e as comparações entre os três grupos foram realizadas pelo teste não paramétrico de Kruskal Wallis com post hoc pelo teste de comparações múltiplas de Dunn. A comparação entre os anos de prática entre os idosos do GCT e GCA foi realizada pelo teste de Mann-Whitney. As correlações entre a idade, a escolaridade e o tempo de prática com as variáveis do MEEM em cada grupo foram realizadas pelos coeficientes de correlação de Spearman. O índice de significância adotado em todas as análises foi de 5\%. Os dados foram analisados pelo pacote estatístico GraphPad versão 5.0 (San Diego - USA).

\section{Resultados}

Os resultados ilustrados na tabela 1 indicam que os idosos apresentaram idades semelhantes e, apesar de os idosos dos três grupos apresentarem baixo nível de escolaridade, o grupo de caratecas apresentou escolaridade dos seus integrantes significativamente maior. Em relação ao tempo de prática, ambos os grupos também apresentaram resultados semelhantes.

Nas variáveis do MEEM, os idosos caratecas (GCT) apresentaram desempenho significativamente melhor quando comparados aos idosos que praticam caminhadas (GCA) e aos não praticantes (GNP). Ao comparar as cinco dimensões avaliadas no instrumento, os grupos se diferiram significativamente somente no aspecto "atenção e cálculo", em que os idosos caratecas apresentaram melhores resultados do que os outros dois grupos. 
Tabela 1 - Resultados descritivos e comparativos entre idade, escolaridade e variáveis do MEEM de indivíduos idosos não praticantes, praticantes de caminhadas e praticantes de caratê.

\begin{tabular}{lcccc}
\hline & GNP & GCA & GCT & p \\
\hline Idade (anos) & $67,5(63-74)$ & $71(64-76)$ & $71(68-75)$ & 0,26 \\
\hline Escolaridade (anos) & $3(1-4)$ & $4((0-4)$ & $4(4-8)$ & $0,04^{*}$ \\
\hline Tempo de prática (anos) & - & $4,5(3-8)$ & $5(3-7)$ & 0,84 \\
\hline MINIMENTAL (pontos) & \multicolumn{5}{c}{} & \\
\hline Orientação & $10(9-10)$ & $9,5(8-10)$ & $10(9-10)$ & 0,55 \\
\hline Memória imediata & $3(3-3)$ & $3(3-3)$ & $3(3-3)$ & 0,76 \\
\hline Atenção e Cálculo & $1(0-3)$ & $2(0-4)$ & $2(2-4)$ & $<0,01^{*}$ \\
\hline Evocação & $2,5(2-3)$ & $2(2-3)$ & $2(2-3)$ & 0,87 \\
\hline Linguagem & $7,5(7,8)$ & $8(6-9)$ & $8,5(8-9)$ & 0,06 \\
\hline TOTAL & $23,5(21-27)$ & $23,5(22-26)$ & $26(24-28)$ & $=0,01^{*}$ \\
\hline
\end{tabular}

* GCT $\neq$ GNP e GCA / GNP: idosos não praticantes de atividades físicas; GCA: idosos praticantes de caminhadas; GCT: idosos praticantes de caratê.

Na tabela 2, em que são apresentados os resultados das correlações entre a idade e o tempo de prática com as variáveis do MEEM em cada grupo, verifica-se correlação significativa negativa com magnitude moderada somente com o escore total do instrumento no GCT. Esse resultado indica que quanto mais novos são os idosos que treinam caratê, melhor é o seu desempenho cognitivo. Essa mesma relação não foi observada em nenhum dos outros dois grupos.

As análises entre a escolaridade e as variáveis do MEEM também mostraram algumas correlações significativas. Na dimensão "atenção e cálculo", os idosos GNP e GCA apresentaram correlações positivas e moderadas, indicando que nesses grupos, os idosos com mais anos de estudo tenderam a realizar de modo mais eficaz as operações matemáticas. Houve também correlação significativa na dimensão "Linguagem" no GCA, no qual o maior tempo de estudo indicou estar associado a um melhor desempenho na verbalização, identificação de objetos, leitura e escrita. Já no escore total do MEEM, correlações significativas foram encontradas entre os grupos GCA e GCT, indicando que esses idosos tenderam a ter melhor pontuação no MEEM, ou seja, melhor estado mental, o qual demonstrou estar diretamente relacionado à quantidade de anos de estudo. 
Tabela 2 - Correlações entre idade e nível de escolaridade com as variáveis do Mini-Exame do Estado Mental nos idosos não treinados, participantes de exercícios generalizados e caratecas.

\begin{tabular}{lllllll}
\hline & \multicolumn{3}{c}{ IDADE } & \multicolumn{3}{c}{ ESCOLARIDADE } \\
\hline & GNP & GCA & GCT & GNP & GCA & GCT \\
\hline Orientação & 0,02 & $-0,46$ & $-0,12$ & 0,10 & 0,30 & 0,25 \\
\hline Memória imediata & 0,00 & 0,28 & $-0,42$ & 0,00 & $-0,04$ & 0,41 \\
\hline Atenção e Cálculo & 0,18 & $-0,11$ & $-0,47$ & $0,57^{*}$ & $0,52^{*}$ & 0,38 \\
\hline Evocação & $-0,43$ & $-0,18$ & $-0,18$ & 0,08 & $-0,38$ & 0,20 \\
\hline Linguagem & $-0,26$ & $-0,08$ & $-0,41$ & 0,38 & $0,55^{*}$ & 0,33 \\
\hline Total & $-0,05$ & $-0,23$ & $-0,66^{* *}$ & 0,39 & $0,63^{* *}$ & $0,60^{* *}$ \\
\hline
\end{tabular}

${ }^{*} p<0,05 ;{ }^{* *} p \leq 0,01$ / GNP: idosos não praticantes de atividades físicas; GCA: idosos praticantes de caminhadas; GCT: idosos praticantes de caratê.

A análise da correlação entre o tempo de prática de atividade física e as variáveis do MEEM dos dois grupos juntos (GCA e GCT) não indicou associação significativa ( $\mathrm{p}>0,05$ para todas), mostrando que o tempo de prática nas atividades investigadas parece não ter interferido sobre o estado mental desses idosos.

\section{Discussão}

O objetivo do estudo foi comparar o estado mental de idosos praticantes de diferentes tipos de atividades físicas. Os resultados demonstraram que o grupo de idosos não praticantes de atividades físicas e o grupo que pratica caminhadas sem supervisão de um profissional possuem desempenho cognitivo abaixo do desejável. Os escores de 23,8 e 23,5, segundo a classificação proposta por Lourenço e Veras (2006), indicam alteração cognitiva em idosos com algum grau de escolaridade e sugerem avaliação cognitiva e funcional mais profunda. Os autores atribuem $24 / 25$ pontos, respectivamente, para o estado cognitivo alterado e para o estado cognitivo normal. Já os idosos caratecas apresentaram escores acima dos 25 pontos, indicando que possuem maiores reservas cognitivas do que os idosos dos outros dois grupos.

A idade, no entanto, não foi um fator influenciador nesses resultados, pois os três grupos tiveram médias semelhantes. A baixa escolaridade apresentada pelos idosos vai ao encontro da realidade da população idosa do Brasil, sobretudo daqueles que residem em cidades de menor porte e do interior do país. Esses idosos, em grande parte, viveram sua infância e juventude 
na zona rural, onde o trabalho no campo e a falta de acesso aos serviços impediram-lhes de frequentar a escola. A escolaridade dos idosos do nosso estudo é coincidente com os resultados encontrados em várias pesquisas realizadas com idosos brasileiros e que também se utilizaram do MEEM(DINIZ; VOLPE; TAVARES, 2007; LOURENÇO; VERAS, 2006; MOTA et al., 2008).

Com relação às comparações entre as categorias do MEEM, os resultados indicaram que na maioria delas, os idosos dos três grupos obtiveram desempenhos semelhantes e dentro do padrão de normalidade. A única categoria que apresentou diferença foi a de "atenção e cálculo", em que os idosos do GCT apresentaram desempenho significativo maior. Observou-se, nessas análises, que os idosos dos grupos GNP e GCA apresentaram dificuldades na realização dessas operações mentais, acertando, em média, menos de dois cálculos dos cinco solicitados no instrumento. Parece que essa categoria apresenta bastante dependência com o nível de escolaridade, pois demanda o uso de operações que são mais frequentemente exigidas no ensino formal.

Assim, o melhor desempenho no MEEM dos idosos do GCT, tanto no escore total quanto na categoria "atenção e cálculo", pode estar relacionado ao maior nível de escolaridade dos seus integrantes e não somente pelo fato de estarem engajados regularmente em atividades de caratê. A literatura específica, tanto nacional como internacional, tem mostrado que o nível de escolaridade é o principal fator influenciador no desempenho cognitivo de idosos (AEVARSSON; SKOOG., 2000; ANDERSON et al., 2007; BERTOLUCCI et al., 1994; BRUCKI et al., 2003).

Apesar dessas evidências, não podemos descartar a possibilidade do programa de caratê ter preservado mais a cognição desses idosos em relação aos outros grupos, uma vez que esse grupo foi o único que participou de atividades sistematizadas e organizadas sob a supervisão de um profissional da área. A modalidade parece solicitar mais dos aspectos cognitivos presentes no instrumento quando comparados aos grupos de caminhada e de não praticantes.

Nos dois últimos grupos, parece não ter tido envolvimento suficiente de operações mentais mais elaboradas para a realização das atividades, tais como atenção, verbalização, decisão, memória, entre outras, as quais são observadas na prática do caratê. Esta inferência é corroborada pelos achados de Man, Tsang e Hui-Chan (2010), que ao investigar três grupos de idosos chineses com níveis de escolaridade semelhantes - praticantes de Tai Chi Chuan, praticantes de exercícios generalizados e não praticantes -, verificaram que os praticantes de Tai Chi apresentaram melhor desempenho cognitivo do que os outros dois grupos. 
Apesar do estudo ser transversal e não ser possível uma relação causal, os autores relacionaram esse resultado ao tipo de atividade praticada, a qual exigia maior atenção e concentração. Embora não tenhamos encontrado estudos que avaliem esses aspectos com idosos praticantes de caratê, acreditamos que este estudo forneça sustentação às nossas hipóteses, pois a prática de Tai Chi Chuan, de maneira semelhante ao caratê, exige condutas de práticas semelhantes nos aspectos de atenção, concentração e complexidade nas ações motoras.

Embora a literatura apresente relação entre a prática de atividade física e a capacidade cognitiva de idosos (KARIMOOY et al., 2012; MUSCARI et al., 2010), não está bem clara a relação com indivíduos que se engajaram mais tardiamente nessas atividades. Geralmente, o que a literatura apresenta sobre esse tema é mais relevante ou significativo quando a prática está presente ao longo da vida do indivíduo. Essa característica não é observada no presente estudo, já que a maioria dos idosos avaliados se engajou sistematicamente em programas de atividade física muito recentemente, isto é, em torno de 4 a 7 anos.

Há outros possíveis aspectos que podem interferir na saúde cognitiva e, consequentemente, no desempenho cognitivo de idosos, tais como a estimulação intelectual, as relações sociais, a linguagem, as habilidades motoras, o planejamento, o exercício da memória e atenção e o nível de atividade física (BUTLER; FORETTE; GREENGROSS, 2004). No presente estudo, foi abordada mais especificamente a influência do efeito da atividade física na capacidade cognitiva de idosos, minimizando os riscos de acometimentos e disfunções mentais.

Middleton et al. (2010) verificaram que a prática de atividade física na adolescência apresentou maior relação no desempenho mental de idosos do que em outras fases da vida e que as mulheres que se tornaram ativas aos 30 ou 50 anos apresentaram melhores resultados nos testes cognitivos realizados durante a velhice. Esses resultados sugerem que quanto mais precoce é o início da prática regular de atividade física, melhores serão os efeitos sobre a cognição dos idosos.

Os resultados referentes às correlações entre a idade e as variáveis do MEEM em cada grupo indicaram correlação significativa somente entre os caratecas no escore total. Esse resultado mostra que a idade parece não exercer grandes influências nos idosos desses grupos avaliados, contrapondo achados de vários estudos que identificaram uma relação negativa entre o aumento da idade e o desempenho no MEEM (DUFOUIL et al., 2000; LAKS et al., 2007; TOMBAUGH; McINTYRE, 1992). 
Esses resultados podem ser explicados pelo fato de os idosos avaliados neste estudo fazerem parte de extratos sociais bem específicos e mais homogêneos, além de serem fisicamente independentes, sem níveis de fragilidades importantes. Essa informação é bastante relevante, já que Auyeung et al. (2011) encontraram resultados confirmando que a idade avançada está correlacionada com o declínio das funções cognitivas em idosos e, sobretudo, em indivíduos fragilizados. Os autores supracitados acompanharam 2.737 idosos durante quatro anos e verificaram que o declínio físico, representado pela diminuição da força e da massa muscular e também da velocidade de marcha, estava relacionado ao declínio cognitivo durante o período do estudo.

No grupo de caratecas, a relação significativa e de magnitude moderada, indicando a tendência de pior desempenho cognitivo com o avançar da idade, pode ser explicada pelo fato desses idosos possuírem maiores escores na pontuação geral do MEEM. O desempenho cognitivo superior pode sofrer maiores influências da idade do que para idosos que apresentaram desempenhos cognitivos inferiores. Isto significa que as pontuações mais baixas no MEEM, inclusive no limiar entre comprometimento e não comprometimento cognitivo, como ocorrido com os idosos dos GNP e GCA, podem ser menos sensíveis à variação da idade.

As correlações entre o estado mental e a escolaridade foram mais significativas do que as com a idade. Conforme o esperado, as correlações significativas foram entre o aspecto "atenção e cálculo" nos grupos GNP e GCT, indicando a dependência do nível de escolaridade para essas operações mentais. De maneira geral, os resultados desse estudo, já discutidos anteriormente, são corroborados pela literatura quanto ao fato do maior nível de escolaridade estar associado ao melhor desempenho cognitivo geral. Essa associação esteve presente em dois dos três grupos avaliados (GNP e GCA). O GCT não apresentou correlações significativas pelo fato de os idosos desse grupo possuírem maior grau de escolaridade do que os dos outros grupos, o que os tornou mais homogêneos em relação a essa variável.

Entendemos como uma das limitações deste estudo o delineamento transversal, o qual não permitiu conclusões em relação à causa e efeito entre as variáveis. Apesar disso, entendemos que estudos preliminares e que avaliem as atividades desenvolvidas na comunidade em situações aplicadas são de extrema importância. Os estudos prospectivos, durante anos de prática - necessários para acompanhar os efeitos da prática de atividades físicas no estado cognitivo de idosos -, são de extrema dificuldade, devido à logística necessária, à adesão à prática e também aos altos custos. 
Outro aspecto de limitação nesse estudo é a amostra reduzida. Por ser o caratê uma modalidade ainda pouco praticada por idosos, os pesquisadores deste estudo encontraram dificuldades em conseguir um número maior de participantes. Apesar dessa limitação, acreditamos na relevância do estudo para que se possa investigar, mesmo que preliminarmente, esse tipo de prática e sua relação com o estado cognitivo de idosos, já que essa modalidade ainda é rara entre idosos brasileiros e envolve condutas e gestos esportivos que precisam ser investigados no contexto do envelhecimento.

\section{Conclusão}

A partir dos resultados encontrados neste estudo, podemos concluir que os idosos praticantes de caratê apresentam melhor desempenho cognitivo do que os idosos que praticam caminhadas sem supervisão de profissional e os não envolvidos em atividades físicas regulares. Além das influências da prática sistematizada e regular do caratê, o maior nível de escolaridade pode ter influenciado no melhor estado mental desses idosos.

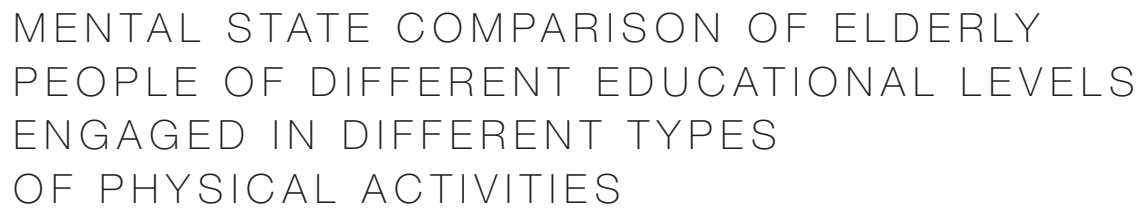

\section{abstract}

The aim of this study was to compare the relation of different types of physical activity with cognitive performance of the elderly. The study included 54 healthy elderly individuals, $70,7( \pm 6,6)$ years old, subdivided into 3 groups: 18 elderly practitioners of karate (GCT), 18 elderly hikers without professional supervision (GCA) and 18 elderly who do not engage regularly in exercise (GNP). The Mini Mental State Examination (MMSE) questionnaire was administered, associating it with the the period of education and involvement in physical exercise. The results showed that on the variables of the MMSE, the elderly karatekas performed significantly better than the elderly who practice hiking and the untrained group. At the MMSE total score, significant correlations were found in the GCT and GCA groups. Thus, we conclude that the elderly tend to have better mental state, by the influence of years of study, as well as by the practice of exercise. 
referências

AEVARSSON, Ólafur; SKOOG, Ingmar. A longitudinal population study of the mini-mental state examination in the very old: relation to dementia and education. Dementia and Geriatric Cognitive Disorders, Basel, v. 11, n. 3, p. 166-175, May/June 2000.

ANDERSON, Tracy M. et al. Effects of sociodemographic and health variables on Mini-Mental State Exam scores in older Australians. The American Joumal of Geriatric Psychiatry, Danvers, v. 15, n. 6, p. 467-476, June 2007.

AUYEUNG, Tung Way et al. Physical frailty predicts future cognitive decline - a four-year prospective study in 2737 cognitively normal older adults. The Journal of nutrition, health and aging, Hong Kong, v. 15, n. 8, p. 690-4, Aug. 2011.

BERTOLUCCI, Paulo Henrique Ferreira et al. Proposta de padronização do Mini-Exame do Estado Mental (MEEM): estudo piloto cooperativo (FMUSP/EPM). Arquivos de Neuropsiquiatria, São Paulo, v. 52, n. 1, p. 01-07, mar. 1994.

BRUCKI, Sonia Maria Dozzi et al. Sugestões para o uso do mini-exame do estado mental no Brasil. Arquivos de Neuropsiquiatria, São Paulo, v. 6, n. 3B, p. 777-781, set. 2003

BUTLER, Robert N.; FOREITE, Françoise; GREENGROSS, Baroness Sally. Maintaining cognitive health in an ageing society. The Journal of the Royal Society for the Promotion of Health, London, v. 124, n. 3, p. 119-121, May 2004.

COLCOMBE, Stanley J. et al. Aerobic fitness reduces brain tissue loss in aging humans. Journal of Gerontology, Washington, v. 58a, n. 2, p. 176-80, Feb. 2003

COSTARELLA, Marianna et al. Decline of physical and cognitive conditions in the elderly measured through the functional reach test and the mini-mental state examination. Archives of gerontology and geriatrics, Amsterdam, v. 50, n. 3, p. 332-337, May/June 2010.

DINIZ, Breno Satler de Oliveira; VOLPE, Fernando Madalena; TAVARES, Almir Ribeiro. Nível educacional e idade no desempenho no Miniexame do Estado Mental em idosos residentes na comunidade. Revista de psiquiatria clínica, São Paulo, v. 34, n. 1, p. 1317,2007

DUFOUIL, Carole et al. Population norms for the MMSE in the very old: estimates based on longitudinal data. Neurology, Minneapolis, v. 55, n. 11, p. 1609-1613, Dec. 2000.

ERICKSON, Kirk I. et al. Exercise training increases size of hippocampus and improves memory. Proceedings of the National Academy of Sciences, Washington, v. 108, n. 7, p. 3017-3022, Feb. 2011.

FOLSTEIN, Marshal F.; FOLSTEIN, Susan; McHUGH, Paul R. Mini-Mental State: a practical method for grading the cognitive state of patients for clinician. Joumal of Psychiatric Research, Amsterdam, v. 12, n. 3, p. 189-198, Nov. 1975.

KARIMOOY, Habibollah Nemati; HOSSEINI, Mahmoud; NEMATI, Maryam. Lifelong physical activity affects mini mental state exam scores in individuals over 55 years of age. Journal of Bodywork \& Movement Therapies, Philadelphia, v. 16, n. 2, p. 230-235, Apr. 2012.

LAKS, Jerson et al. Mini-Mental State Examination norms in a community dwelling sample of elderly with low schooling in Brazil. Cadernos de Saúde Pública, São Paulo, v. 23, n. 2, p. 315-319, Feb. 2007. 
LOURENÇO, Roberto A; Veras, Renato P. Mini-Exame do Estado Mental: características psicométricas em idosos ambulatoriais. Revista de Saúde Pública, São Paulo, v. 40, n. 4, p. 712-719, Ago. 2006.

MAN, David W; TSANG, William W; HUI-CHAN, Christina W. Y. Do older tai chi practitioners have better attention and memory function? Joumal of Alternative and Complementary Medicine, New York, v. 16, n. 12, p. 1259-1264, Dec. 2010.

MIDDLETON, Laura E. et al. Physical Activity Over the Life Course and Its Association with Cognitive Performance and Impairment in Old Age. Journal of the American Geriatrics Society, Los Angeles, v. 58, n. 7, p. 1322-1326, July 2010.

MOTA, Márcia Maria Peruzzi E. et al. Triagem cognitiva: comparações entre o minimental e o teste de trilhas. Estudos de Psicologia, Campinas, v. 25, n. 3, p. 353-359, jul./set. 2008.

MUSCARI, Antonio et al. Chronic endurance exercise training prevents aging-related cognitive decline in healthy older adults: a randomized controlled trial. International Journal of Geriatric and Psychiatry, New York, v. 25, n. 10, p. 1055-1064, Oct. 2010.

PEREIRA, Silvia Regina Mendes et al. Quedas em idosos. In: JATENE F.B. et al. (Orgs). Projeto diretrizes. São Paulo: Associação Médica Brasileira, 2002. p. 405-414.

PSALTOPOULOU, Theodora et al. Diet, physical activity and cognitive impairment among elders: the EPIC - Greece cohort (European Prospective Investigation into Cancer and Nutrition). Public Health Nutrition, Cambridge, v. 11, n. 10, p. 1054-1062, Oct. 2008 .

RIIKLI, Roberta E.; EDWARDS, Darren J. Effects of a three-year exercise program on motor function and cognitive processing speed in older women. Research Quarterly for Exercise and Sport, Washington, v. 62, n. 1, p. 61-67, Mar. 1991.

SANTOS, Diana; XAVIER, André Junqueira; MONTE, Fernanda Guidarini; PARCIA, Silvia Rosane. Aptidão Cardiorrespiratória e função cognitiva em idosos. Estudos Interdisciplinares sobre o envelhecimento, Porto Alegre, v. 16, n. 1, p. 127-142, jun. 2011.

SPIRDUSO, Waneen Wyrick. Reaction and movement time as a function of age and physical activity level. Journal of Gerontology, Washington, v. 30, n. 4, p. 435-440, July 1975.

STARKES, Janet L. et al. Aging and the retention of sport expertise. International Journal of Psychology, Amsterdam, v. 30, n. 2, p. 283-301, 1999.

TOMBAUGH, Tom N.; Mclntyre, Nancy J. The mini-mental state examination: a comprehensive review. Joumal of the American Geriatrics Society, New York, v. 40, n. 9, p. 922-935, Sep. 1992.

ZISI, Vasiliki et al. Effects of a short-term exercise program on motor function and whole body reaction time in the elderly. Joumal of Human Movement Studies, Londres, v. 40, p. 145-160, 2001. 